Edge kink/ballooning mode stability in tokamaks with separatrix

This article has been downloaded from IOPscience. Please scroll down to see the full text article.

2006 Plasma Phys. Control. Fusion 48927

(http://iopscience.iop.org/0741-3335/48/7/003)

The Table of Contents and more related content is available

Download details:

IP Address: 128.178.125.35

The article was downloaded on 08/04/2010 at 16:22

Please note that terms and conditions apply. 


\title{
Edge kink/ballooning mode stability in tokamaks with separatrix
}

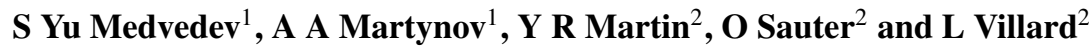 \\ ${ }^{1}$ Keldysh Institute for Applied Mathematics, Russian Academy of Sciences, Moscow, Russia \\ ${ }^{2}$ CRPP, Association Euratom-Confédération Suisse, EPFL, Lausanne, Switzerland \\ E-mail: medvedev@a5.kiam.ru
}

Received 16 February 2006

Published 25 May 2006

Online at stacks.iop.org/PPCF/48/927

\begin{abstract}
Stability limits against external kink modes driven by large current density and pressure gradient values in the pedestal region are investigated for tokamak plasmas with separatrix. Stability diagrams for modes with different toroidal wave numbers under variations of pressure gradient and current density in the pedestal region are presented for several equilibrium configurations related to TCV. A scaling for the toroidal wave number of the most unstable mode is proposed. The influence of the plasma cross-section geometry on the stability limits is discussed.
\end{abstract}

(Some figures in this article are in colour only in the electronic version)

\section{Introduction}

The stability limits of the pedestal or edge region of tokamak plasmas is an important issue for understanding the edge localized mode (ELM) behaviour and optimization of large tokamaks like ITER. The corresponding stability limits provide an input to nonlinear models of ELM dynamics and useful insights for the integrated modelling of ELMs and their control in tokamaks.

The ideal MHD theory identifies the instabilities that trigger the ELMs as coupled peelingballooning modes or, more generally, current and pressure driven modes due to the large values of current density and pressure gradient in the pedestal region $[1,2]$.

The differences between limiter and separatrix geometries relevant to the peeling mode stability analysis were listed in [1]; because the safety factor goes to infinity at the separatrix, there are resonant surfaces inside the plasma for any mode number. Moreover, there is a non-negligible variation of $q$ across the mode width. The question of the applicability of the localized peeling mode criterion [3,4] to the ideal divertor plasma still needs to be answered. Plasma outside the separatrix and the possible transition of the peeling mode into resistive tearing mode should also be taken into account. The edge kink/ballooning mode is a more robust pressure-driven instability which can set the stability limit lower than the $n=\infty$ 
ballooning modes due to coupling with current driven external modes. It decouples from the localized peeling mode in a limiter plasma when the closest resonant surface in vacuum is far from the plasma so that the edge value of $m-n q \sim 1$. With the separatrix at the plasma boundary the edge kink/ballooning mode gives stability limits which do not depend on small variations of the safety factor. As a result, a clearer picture of the edge instabilities arises. In particular, clarifying scalings and stability limits can be established [5].

The infinite safety factor at the plasma boundary is an essential difficulty also for numerical modelling of the separatrix plasma. However, in finite element codes like KINX [6] there is a possibility of modelling the separatrix stabilizing influence without excessive resolution near the plasma boundary. It is based on the ideal stability formulation using $\xi \cdot \nabla \psi$ as one of the unknown projections of the plasma displacement $\xi$. Taking into account that the equilibrium poloidal flux gradient $\nabla \psi$ vanishes at the x-point gives a regularity condition there: $\xi \cdot \nabla \psi=0$.

A useful option for edge stability analysis is setting the plasma displacement to zero inside a prescribed magnetic surface and solving only for displacements in the rest of the plasma. It provides the possibility of increasing the radial resolution near the plasma edge.

The last version of the KINX stability code was also upgraded to compute high- $n$ mode stability. A new variant of the ballooning factor extraction is applicable to equilibria with separatrix and significantly enhances the grid convergence in the case of high toroidal wave numbers especially for large $q$ variations. The details of the implementation are given in the appendices.

\section{A $w \times n \times q_{95}=$ const scaling for edge instabilities}

A scaling for the toroidal mode numbers of the current driven edge instabilities was proposed in [5]. In the parametric plane given by the values of pressure gradient and current density in the pedestal, the stability boundaries for modes with the same product $w \times n$ of the pedestal width $w$ and toroidal mode number $n$ were found to be very close to each other. The scaling can be generalized to the form $w \times n \times q_{95}=$ const under variations of the total plasma current, where $q_{95}$ is the value of the safety factor at $95 \%$ of the poloidal flux inside the separatrix. Let us note that the scaling is applicable to the modes localized in the edge region, i.e. of radial extent that is comparable to or slightly larger than the pedestal width. The intermediate-n mode localization near the plasma edge (typically $0.7<\psi / \psi_{\text {edge }}<1$ that corresponds to $0.84<\sqrt{\psi / \psi_{\text {edge }}}<1$ ) was also demonstrated in [7]. A coupling to the plasma core can significantly change the stability boundaries for the global modes, especially for $n=1$.

The scaling can provide a prediction of the most unstable mode number once the stability boundaries are known for specific pedestal shape and equilibrium profiles. Another important parameter that can be predicted is the critical value of the current density in the pedestal region. The ratio of the maximal parallel current density in the pedestal region $J_{\|}=\max _{\text {pedestal }}\langle j B\rangle_{\psi} /\langle|B|\rangle_{\psi}$ (where $\langle\cdot\rangle_{\psi}=(\mathrm{d} / \mathrm{d} \psi) \int_{V_{\psi}} \cdot \mathrm{d} V /(\mathrm{d} / \mathrm{d} \psi) V_{\psi}$ is the flux surface average over the volume between magnetic surfaces, with $V_{\psi}$ being the volume inside the surface with the poloidal flux $\psi)$ to the averaged plasma current density $\langle J\rangle=I_{p} / S_{p}\left(I_{p}\right.$ and $S_{p}$ being the plasma current and toroidal cross-section) provides a suitable measure for that. $J_{\|} /\langle J\rangle \sim 1$ is a good estimate for the limit against current driven (not peeling!) modes almost independent of the pedestal width $w$. The pedestal width $w$ determines the most unstable toroidal mode number $n$ according to the scaling $w \times n=$ const.

The current limit with the separatrix at the boundary, appears to have a close relation to the instability condition $J_{\|} /\langle J\rangle>1$ [1] for the circular plasma with $m-n q=1$ at the boundary, i.e. when the resonant surface in vacuum is far from the plasma. High values of 
$J_{\|} /\langle J\rangle$ result in shear reversal near the plasma edge. For a large aspect ratio and circular cross section plasma $J_{\|} /\langle J\rangle=(2-S) / 2$, where $S=(r / q) \mathrm{d} q / \mathrm{d} r$ is the shear, so the shear reversal and current limits coincide. Shaping of the plasma cross section, in particular the triangularity, increases the threshold value of $J_{\|} /\langle J\rangle$ necessary to reach the shear reversal. The presence of the separatrix at the plasma boundary further increases the threshold, especially when the local maximum of the parallel current density in the pedestal is located at the separatrix. So the edge current limit $J_{\|} /\langle J\rangle=1$ is not directly connected to the shear reversal. However, non-monotonic safety factor profile leads to coupling of external kink modes to infernal-type modes strongly destabilized by finite pressure gradient in the low shear region.

There is also a dependence on the value of the parallel current density at the plasma vacuum interface $J_{\text {edge }} /\langle J\rangle$ that is chosen as an analogous critical parameter in [8] (with the ratio $J_{\text {edge }} / J_{\|} \sim 1 / 6$ nearly fixed). The ratio $J_{\|} /\langle J\rangle$, with $J_{\|}$being the maximal value of pedestal parallel current density, better quantifies the stability boundaries: critical values of $J_{\|} /\langle J\rangle$ vary moderately under quite strong variations of the ratio $J_{\text {edge }} / J_{\|}$. Moreover, the shear reversal is related to the maximal value of the current density rather than to the edge value. Destabilization of coupled external kink/infernal modes due to non-monotonic safety factor profile in the pedestal sets the current limit corresponding to the shear reversal even for $J_{\text {edge }}=0$. The change in the $J_{\text {edge }} / J_{\|}$value can lead also to a shift in the most unstable toroidal wave numbers [5]. In other words, the value of the constant in the scaling $w \times n \times q_{95}=$ const depends on the $J_{\text {edge }} / J_{\|}$ratio. Of course the coupling to ballooning modes can reduce the stability threshold $J_{\|} /\langle J\rangle$.

In the TCV tokamak, stationary ELMy H-modes are routinely obtained, without additional heating, in single null diverted plasmas with $I_{p}>400 \mathrm{kA}$ and $q_{95} \sim 2.5$. A magnetic perturbation was applied to the plasma vertical position control feedback loop to induce a vertical excursion. These movements led to changes in the edge current density which in turn destabilize the ELMs. Since ELMs were found to synchronize on the perturbation, ELM frequency was then controllable over a range around the natural ELM frequency [9]. The reconstructed equilibrium of one discharge (\#20333) used in these experiments, with the separatrix at the boundary, was a starting point for the stability analysis.

The following procedure was proposed for the stability diagram generation.

- Use experimental data to reconstruct an equilibrium. Note that the accuracy of the profiles in the pedestal region is unfortunately not always sufficient.

- Change independently the current density and pressure gradient profiles in the pedestal region by adding the following function multiplied by a constant to the original reconstructed ones (parallel current density $\langle j B\rangle_{\psi} /\langle B \nabla \phi\rangle_{\psi}$ and pressure gradient $p^{\prime}=$ $\mathrm{d} p / \mathrm{d} \psi)\left(\right.$ figure 1): $1-\tanh ^{2}\left(\frac{x_{0}-s}{w}\right)$, where $s$ is the square root of normalized poloidal flux, keeping the normalized current $I_{N}=I_{p}[M A] /\left(a[m] B_{c}[T]\right)$ fixed. Recompute consistent equilibria.

- Compute the stability boundaries for different mode numbers $n$ for the obtained equilibria.

The width of the pedestal $w$ and the location of the pedestal $x_{0}$ (leading to different current density values at the separatrix) are independent parameters in the study.

The first series of computations with $x_{0}=1$ corresponds to the maxima of pressure gradient $p^{\prime}$ and current density $J_{\|}$located at the separatrix. The results are summarized in figure 2. Here the stability boundaries are given for three values of the pedestal width $w$ and different toroidal wave numbers $n$. The marginally stable value of edge current density is almost constant with increasing edge pressure gradient up to the ballooning limit in the first stability region at the separatrix $p^{\prime}=p_{c}^{\prime}$ and then decreases with increasing $p^{\prime}$. There 

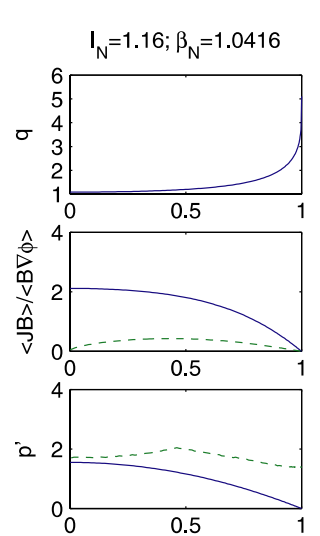

a) $\operatorname{sqrt}(\psi)$
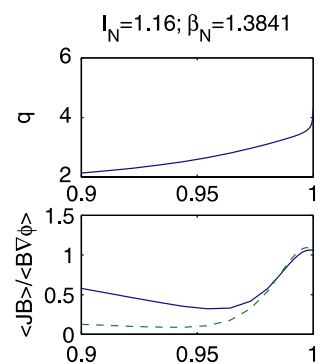

-

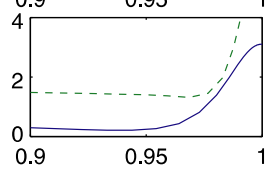

b)
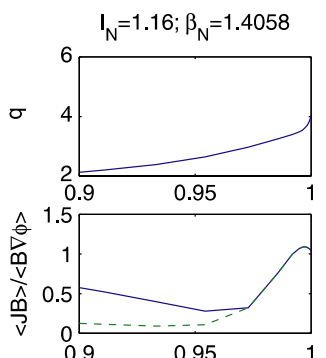

○

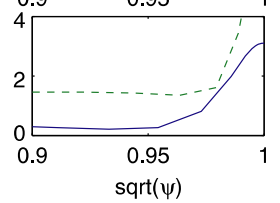

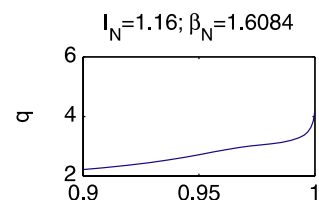

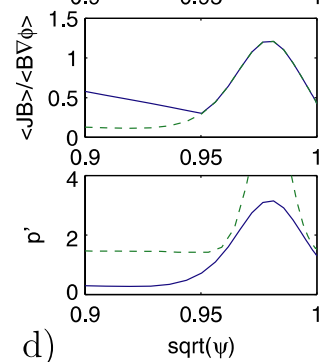

Figure 1. Profiles of the TCV shot \#20333 reconstructed equilibria ( $a$ ) and zoomed region near the boundary with different pedestal profiles corresponding to prescribed $(b)\left(p^{\prime} / p_{c}^{\prime}=2.22, J_{\|} /\langle J\rangle=\right.$ 1.05) and self-consistent bootstrap $(c),(d)\left(p^{\prime} / p_{c}^{\prime}=2.22\right)$ current density. The bootstrap current and marginally ballooning stable pressure gradients are shown by dashed lines.

are no signs of second stability access with increasing $n$ up to 60 ; the stability region in the $\left(p^{\prime} / p_{c}^{\prime}, J_{\|} /\langle J\rangle\right)$ plane just slightly shrinks with increasing toroidal wave number. One can also notice that the stability boundaries for different pedestal width $w$ and different $n$ but with the same product $w \times n$ are close to each other. The dashed line in figure 2 indicates the edge bootstrap current density computed in the collisionless limit [10]. The dotted line in figure 2 indicates the stability limit given by the peeling mode criterion $[3,4]$, which is very clearly different from the stability boundaries consistently computed with the separatrix at the plasma boundary. The structure of a typical current driven kink mode is shown in figure 3 .

The situation changes when the pedestal is inside the plasma $\left(x_{0}<1\right)$ and $J_{\text {edge }}$ is lower than its maximal value in the pedestal region near $x_{0}$. There is a second stability access for the modes with $n \gtrsim 20$ in the case with $x_{0}=0.98, w=0.02$ (figure 4 ). The structure of the coupled kink-ballooning mode is shown in figure 5. Another consequence of moving the pedestal position inside the plasma is the occurrence of shear reversal for lower values of normalized current density in the pedestal leading to coupled current driven and infernal mode destabilisation; the corresponding line above which this happens is indicated in figure 4 .

The shape of the pedestal can be defined by the ratio of the edge current density and pressure gradient to their maximums in the pedestal. The tables below illustrate the $w \times n=$ const scaling under variations of the pedestal width for different pedestal shapes that determine a value of the constant in the scaling. Series of equilibria with self-consistent bootstrap current in the collisionless limit [10] were used to demonstrate this. Marginal values of the pedestal pressure gradient $p^{\prime} / p_{c}^{\prime}$ with the corresponding values of $J_{\|} /\langle J\rangle$ are given for different values of toroidal mode number $n$ for $p_{\text {edge }}^{\prime} / p^{\prime}=0.42$ and two values of the pedestal width parameter $w$.

\begin{tabular}{llllll}
\hline \multicolumn{2}{c}{$x_{0}=0.98, w=0.02$} & \multicolumn{3}{c}{$x_{0}=0.99, w=0.01$} \\
\hline$n$ & $p^{\prime} / p_{c}^{\prime}$ & $J_{\|} /\langle J\rangle$ & $n$ & $p^{\prime} / p_{c}^{\prime}$ & $J_{\|} /\langle J\rangle$ \\
10 & 1.8056 & 0.91 & 20 & 1.7959 & 0.91 \\
15 & 1.7413 & 0.88 & 30 & 1.7448 & 0.89 \\
20 & 1.7393 & 0.88 & 40 & 1.7778 & 0.90 \\
\hline
\end{tabular}




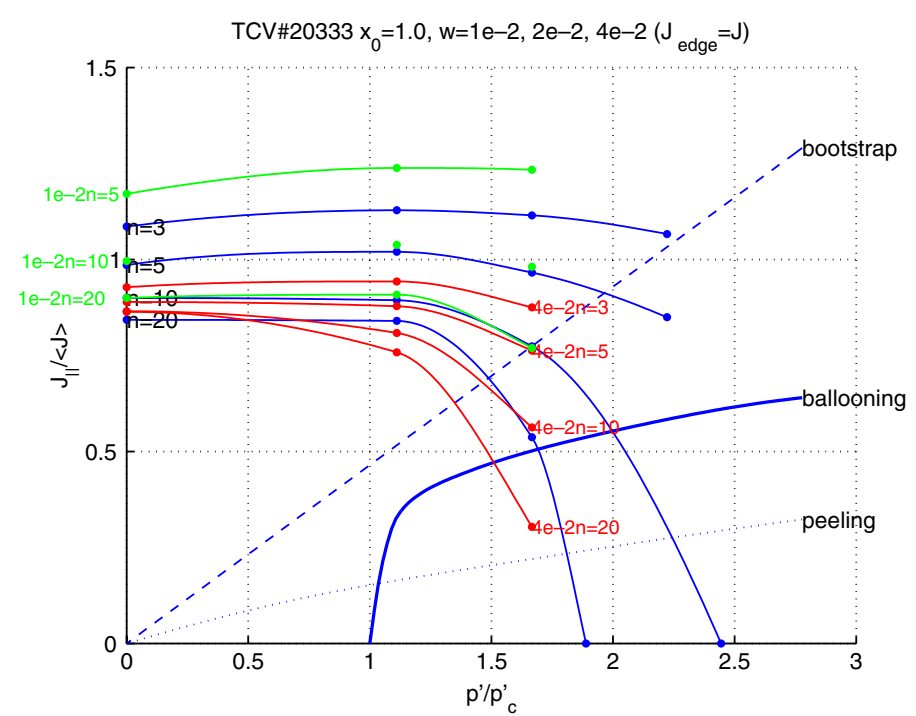

Figure 2. Stability diagrams for the case with the pedestal at the separatrix for the TCV shot \#20333. Colours of the curves correspond to different pedestal widths $w$ : 0.01 (green), 0.02 (blue), and 0.04 (red). The curves are labelled by toroidal modes numbers and the $w$ values (1e-2 and 1e-4). The thick line shows $n=\infty$ ballooning mode stability boundaries. Dashed line-bootstrap current density, dotted line-localized peeling mode stability boundary. The modes are stable below the corresponding curves (to the left in the case of second stability access).

The next table corresponds to $p_{\text {edge }}^{\prime} / p^{\prime}=0.18$ and shows that the range of most unstable modes shifts to lower values of $n$ with the pedestal moving deeper into the plasma (in other words, the second stability access takes place for lower values of $n$ ).

\begin{tabular}{llllll}
\hline \multicolumn{3}{c}{$x_{0}=0.97, w=0.02$} & \multicolumn{3}{c}{$x_{0}=0.985, w=0.01$} \\
\hline$n$ & $p^{\prime} / p_{c}^{\prime}$ & $J_{\|} /\langle J\rangle$ & $n$ & $p^{\prime} / p_{c}^{\prime}$ & $J_{\|} /\langle J\rangle$ \\
5 & 2.4111 & 1.17 & 10 & 2.3320 & 1.13 \\
10 & 2.1725 & 1.07 & 20 & 2.2222 & 1.09 \\
15 & 2.2222 & 1.09 & 30 & 2.3597 & 1.15 \\
\hline
\end{tabular}

The stability diagrams for the cases with narrower pedestal and lower current density at the separatrix are presented in figure 6. A comparison of diagrams in figures $6(a)$ and figure 4 shows the applicability of the scaling $w \times n=$ const to the stability boundaries in the parametric space for modes with corresponding wave numbers. Besides the shift to lower values of the toroidal wave numbers mentioned above, the lower edge current density significantly increases the maximal attainable pressure gradient in the pedestal due to the enhanced access to the second stability region (figure $6(b)$ ). Due to lower current density at the separatrix current driven modes become unstable at higher values of $J_{\|} /\langle J\rangle$ which give shear reversal near the edge. Figure 7 shows the structure of the current driven mode $n=1$ for the equilibrium with the value of $J_{\|} /\langle J\rangle=1.35$ near the stability margin at low values of pressure gradient in the pedestal (shear reversal takes place at $J_{\|} /\langle J\rangle=1.3$ ). The profile of the $m=3$ poloidal harmonic exhibits a maximum in the region with low shear close to the local $q$ extremums close to the rational surface $q=3$ that is typical of infernal mode structure. 

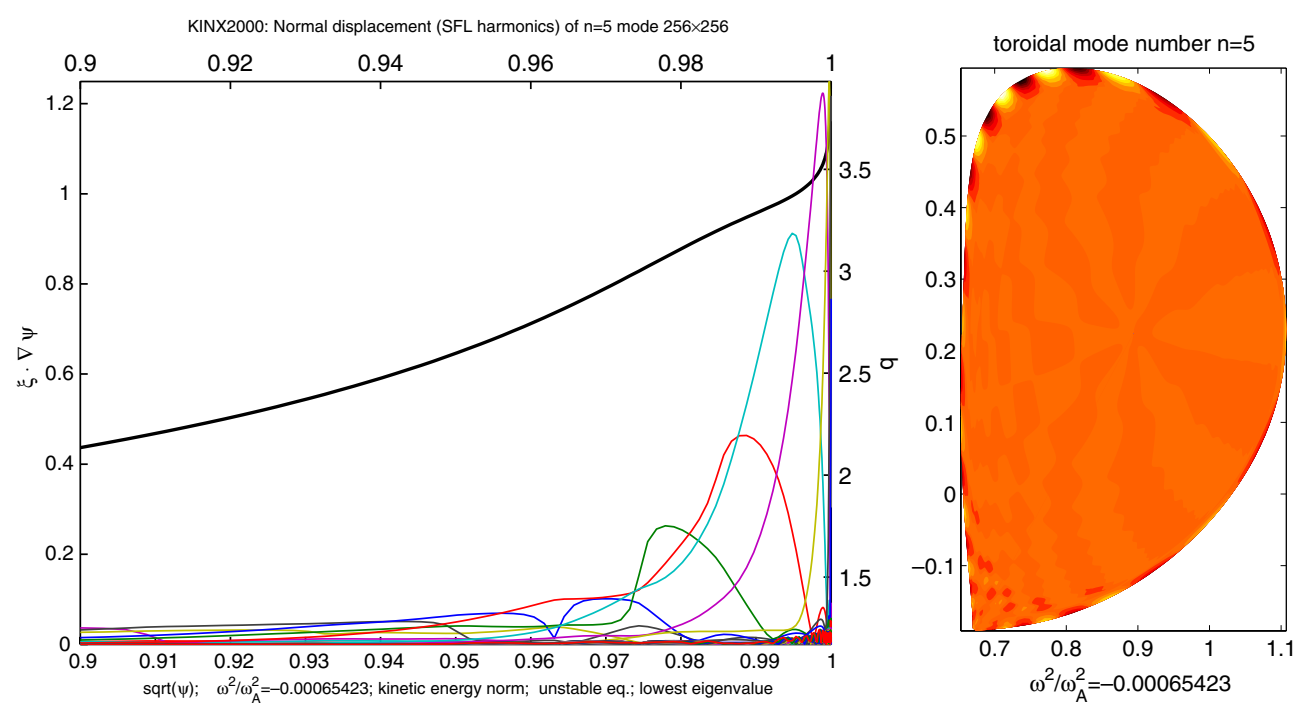

Figure 3. Current driven mode structure. Amplitudes of harmonics in straight magnetic field line poloidal angle and contour plot for the normal displacement of the unstable eigenfunction. The $q$-profile is shown in bold line. Equilibrium corresponding to the TCV shot \#20333 with current density spike at the separatrix $\left(x_{0}=1.0, w=0.02, p^{\prime} / p_{c}^{\prime}=0, J_{\|} /\langle J\rangle=1.0\right)$. Toroidal wave number $n=5$.

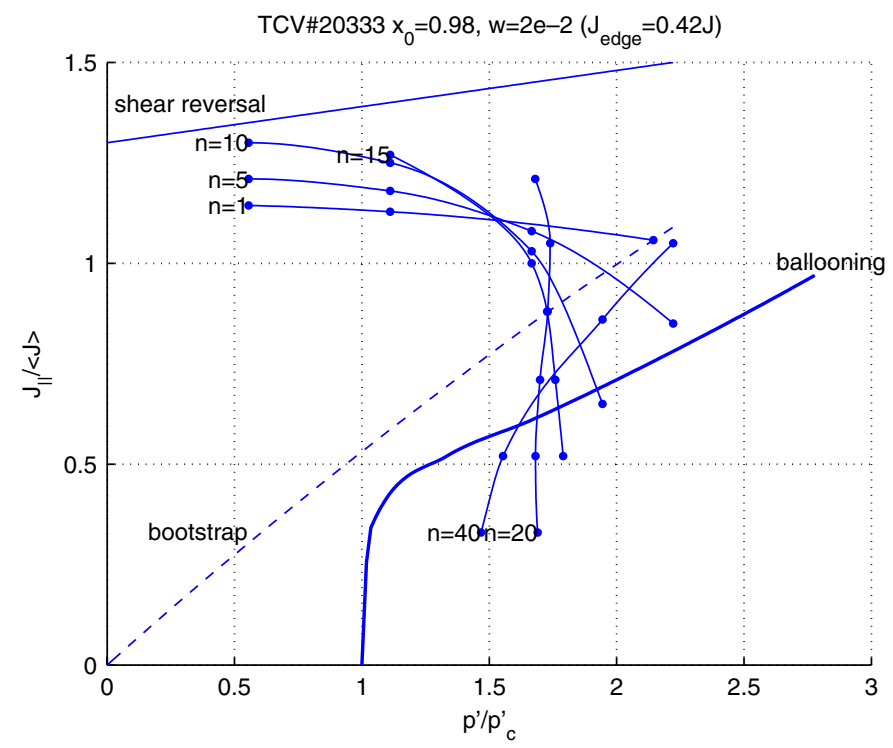

Figure 4. Stability diagrams for the case with the pedestal inside the plasma for TCV\#20333. An example of the corresponding profiles is given in figure $1(d)$.

A higher- $q$ equilibrium with the same boundary and profiles of parallel current density and pressure gradient was obtained by increasing the toroidal magnetic field to get a normalized toroidal current value $I_{N}=0.58$ twice as low as that in the reconstructed equilibrium. The stability diagrams for that case are presented in figure $8(a)$. From the comparison with the 

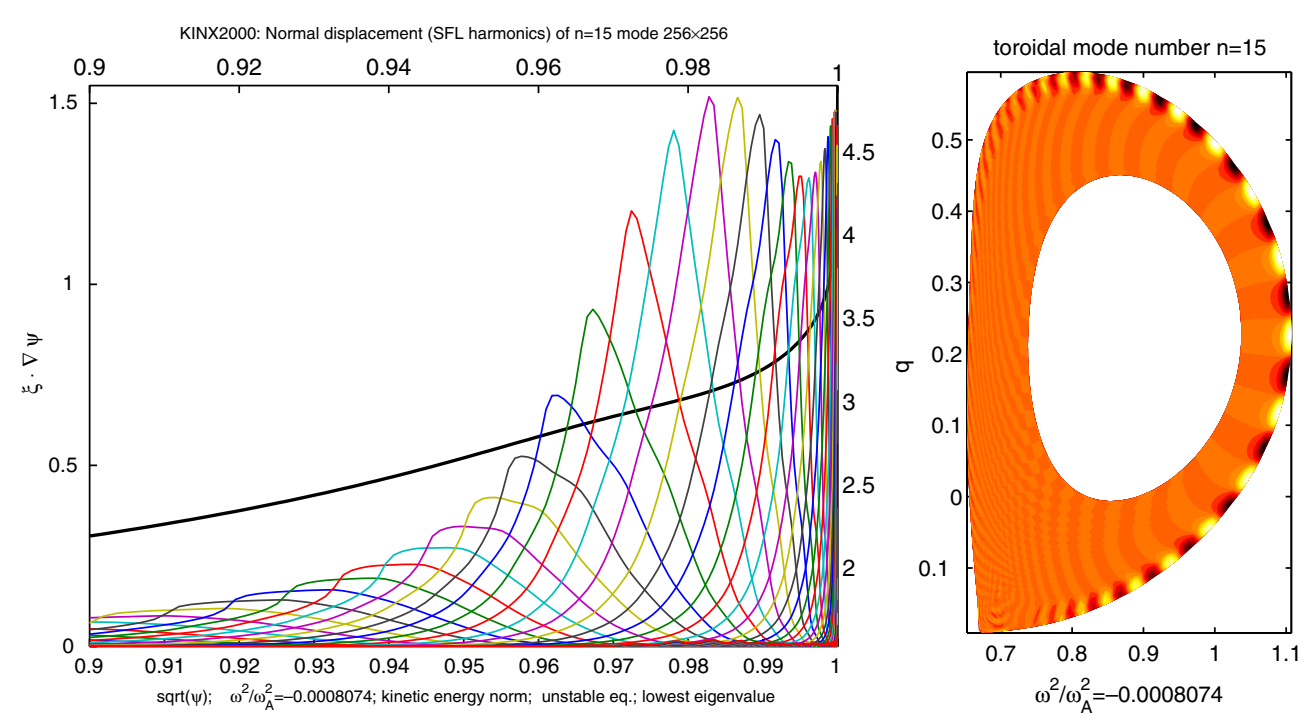

Figure 5. Kink/ballooning mode structure. Equilibrium corresponding to TCV \#20333 with pedestal inside the plasma $\left(x_{0}=0.98, w=0.02, p^{\prime} / p_{c}^{\prime}=1.8, J_{\|} /\langle J\rangle=0.9\right)$. Toroidal wave number $n=15$.
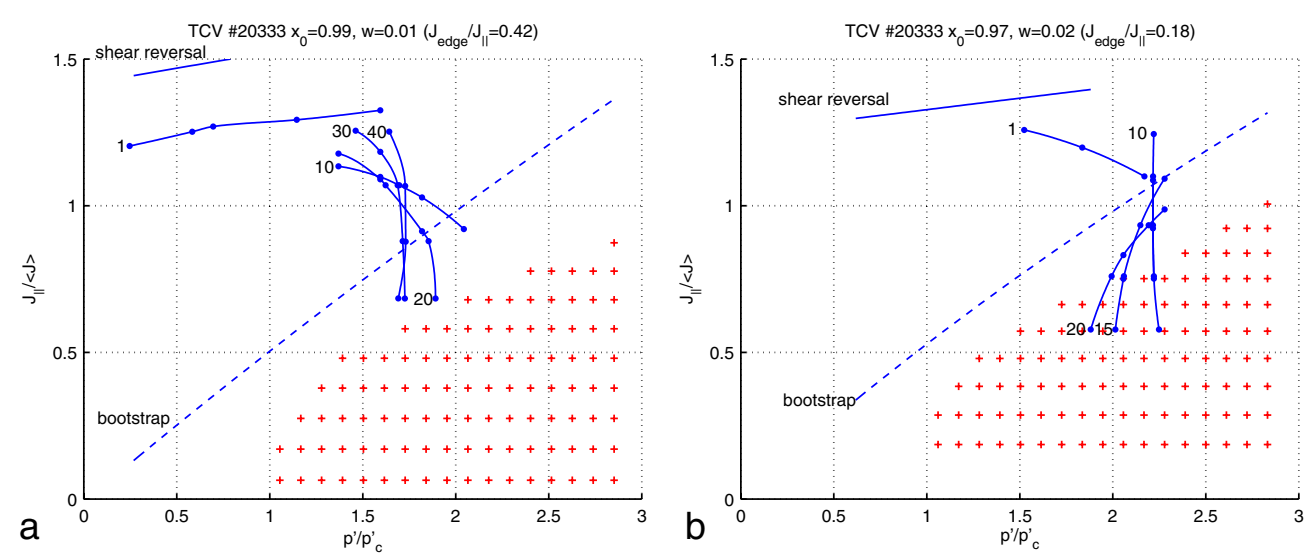

Figure 6. Stability diagrams for the case of narrower pedestal width $((a)$ left) and for the case of lower current density at the separatrix $((b)$ right $)$. High- $n$ ballooning unstable region is marked by crosses.

diagrams from figure 4 one can note that the scaling $w \times n \times q_{95}=$ const holds for the toroidal wave number of the most unstable mode limiting the pressure gradient: in particular, $n=10$ and $n=20$ modes, respectively, limit the access to high $p^{\prime}$ for equilibria with different $q_{95}$ with nearly bootstrap aligned current density in the pedestal. An important trend is the increased distance between the bootstrap line and the high- $n$ ballooning mode stability boundary in the parametric plane. This is due to the lower current density needed for second stability access at higher $q$. The value of $p_{c}^{\prime}$ at the separatrix is higher for the configuration with higher $q_{95}$. Higher $l_{i}$ equilibrium with different profiles but with the same normalized current value $I_{N}=0.58$ and $q_{0}=1.1$ close to the value in the reconstructed equilibrium was also considered (the corresponding value of the $q_{95}$ increased from 2.75 to 5.5). The stability diagrams are 

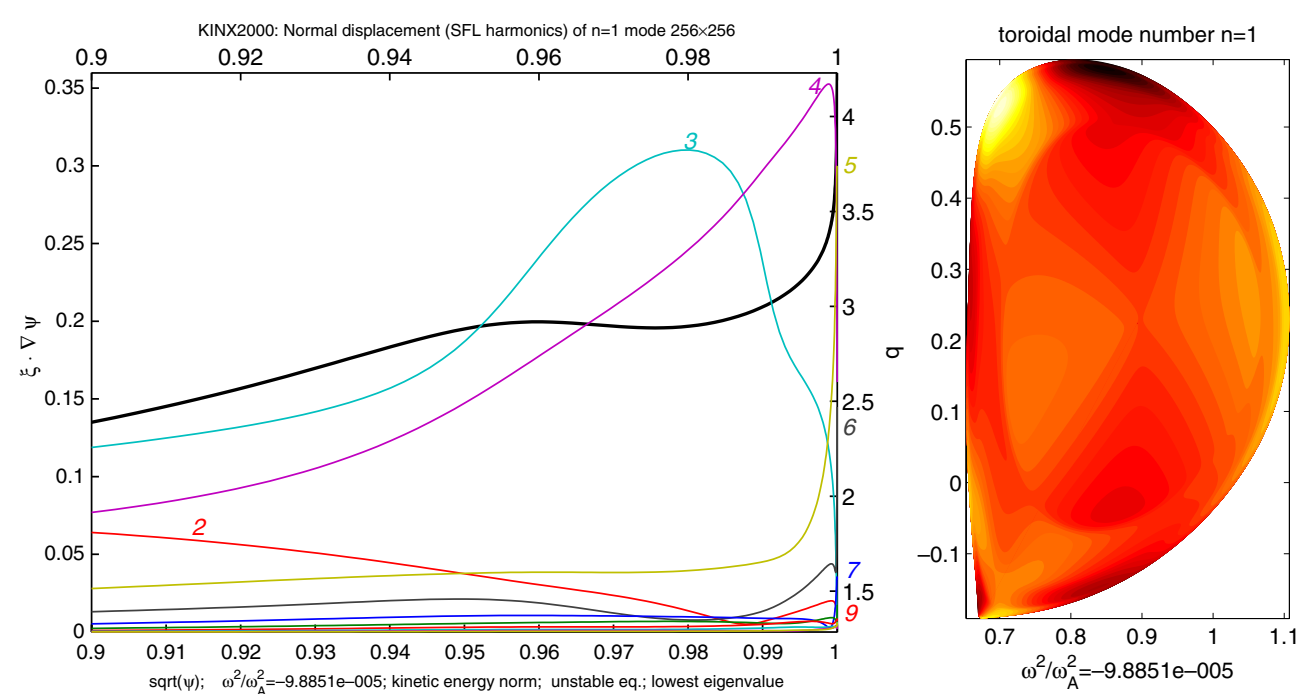

Figure 7. Current driven mode structure. Equilibrium with lower current density at the separatrix and shear reversal near the edge. TCV \#20333 plasma shape $\left(x_{0}=0.97, w=0.02\right.$, low $p^{\prime} / p_{c}^{\prime}$, $J_{\|} /\langle J\rangle=1.4$ ). Toroidal wave number $n=1$. Poloidal mode numbers are shown for dominating harmonics.
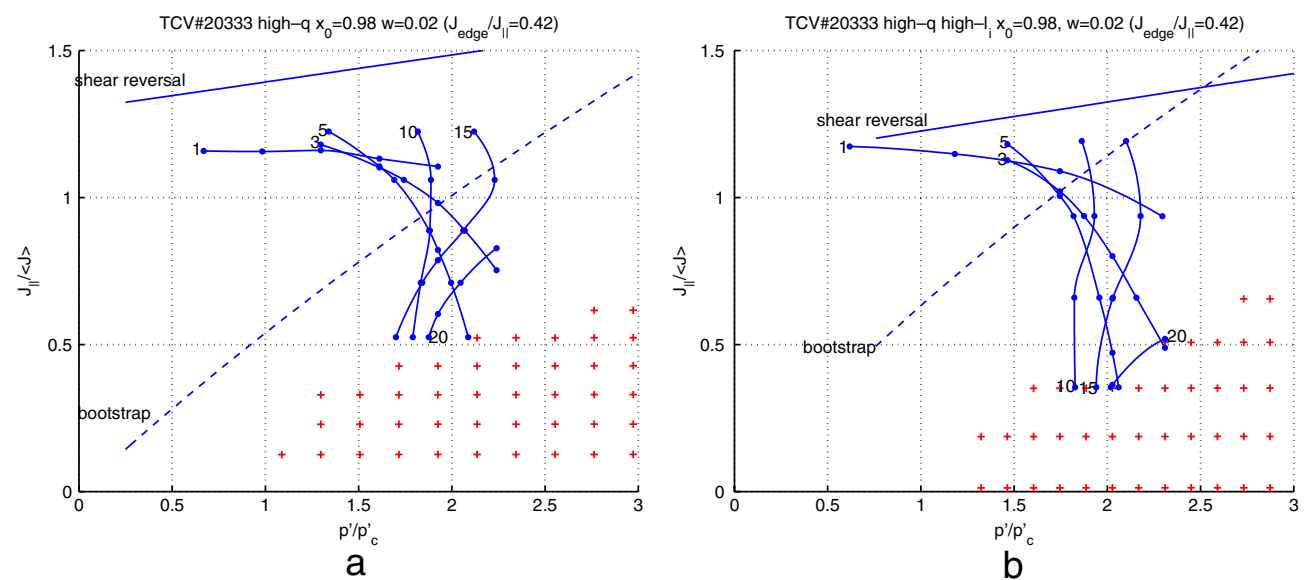

Figure 8. Stability diagrams for the high- $q((a)$ left $)$ and high- $q$ higher $l_{i}((b)$ right $)$ cases.

close to the previous high- $q$ case, at least for medium $n<15$ (figure $8(b)$ ). The value of $p_{c}^{\prime}$ at the separatrix is somewhat lower for this configuration with a higher boundary shear but the second stability access takes place for lower values of normalized current. That behaviour is reflected by the $n=20$ mode stable for lower values of $J_{\|} /\langle J\rangle$.

\section{Plasma shape influence}

Higher-order moments of the plasma cross-section-triangularity and squareness-play an important role in defining the limits for both high- $n$ ballooning and kink/ballooning modes [12]. 


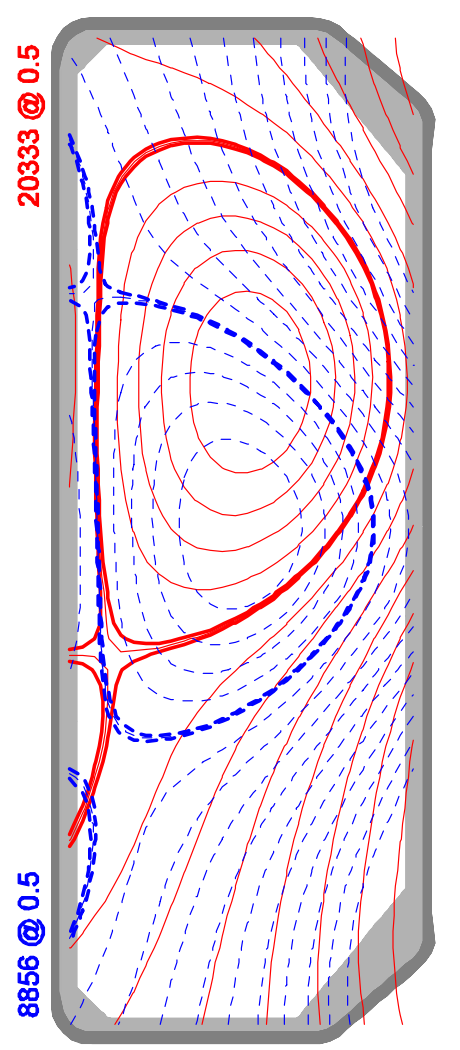

Figure 9. Magnetic surfaces for TCV shots \#20333 (red, solid lines) and \#8856 (blue, dashed lines) and the TCV vacuum chamber.

In TCV, allowing an extreme flexibility in the plasma shaping [13], ELMs are obtained in a wide variety of plasma shapes. For instance, discharge \#8856 also showed ELMs although at a less regular frequency. In this discharge, the plasma elongation was much reduced while the triangularity was increased $(\delta=0.58)$ in such a way that the configuration became double null (figure 9). This discharge was then chosen for the comparison.

The first unexpected feature for this configuration is the proximity of the bootstrap line and high- $n$ ballooning stability limit in the parametric plane (figure $10(a)$ ). The reason for this can be the lower elongation $(E=1.6)$ and lower squareness of the plasma cross section that affects the high- $n$ ballooning mode stability, especially near the separatrix. Probably the same is true for medium- $n$ kink/ballooning stability boundaries: despite the larger value of triangularity the stability diagrams resemble those for the shot \#20333 ( $\delta_{\text {up }}=0.36$ ) with the mode $n=20$ closing the way to high $p^{\prime} / p_{c}^{\prime} \gtrsim 1.5$ and being marginal for the second stability access. A quite low stable value of $J_{\|} /\langle J\rangle<0.9$ limited by the global $n=1$ kink mode is due to the lower value of internal inductance $l_{i}=0.6$ ( $l_{i}=0.76$ for the \#20333 configuration). The structure of unstable modes in the region $J_{\|}<J_{\text {bootstrap }}$ is dominated by the ballooning component (figure 11).

Increasing the elongation by stretching the cross-section to $E=2$ makes it closer to the ITER configuration [11] but having the $\mathrm{x}$-point at the plasma top with the corresponding shift in the medium-n kink/ballooning limit to $p^{\prime} / p_{c}^{\prime} \sim 2.5$ (figure $10(b)$ ). 

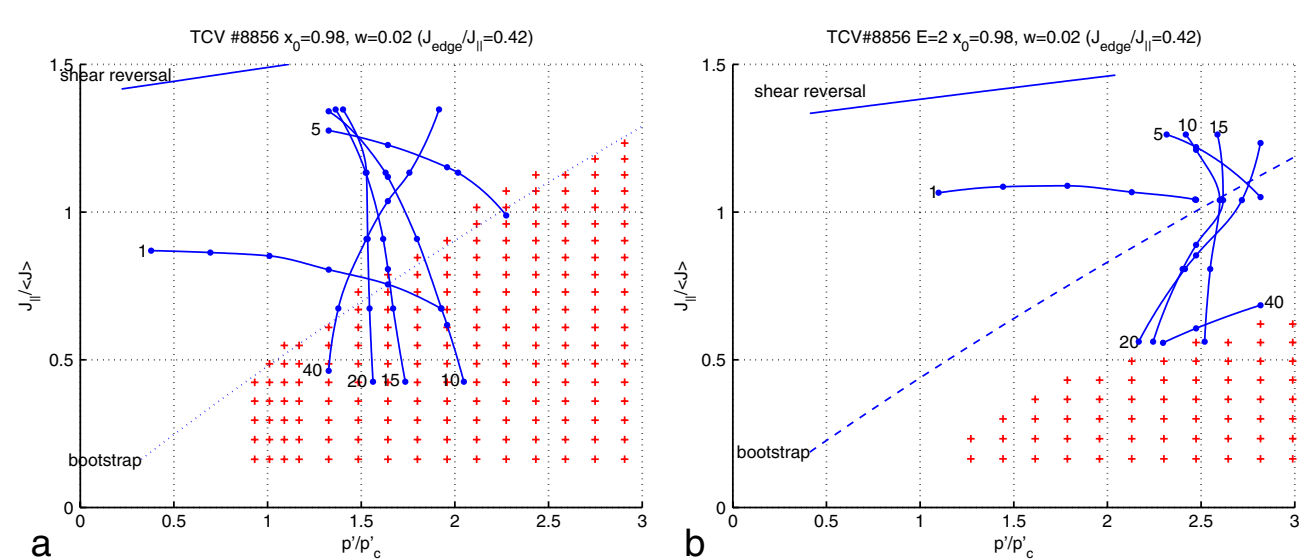

Figure 10. Stability diagrams for the TCV shot \#8856 case. The original elongation $E=1.6((a)$ left) and the increased elongation $E=2$ ((b) right).
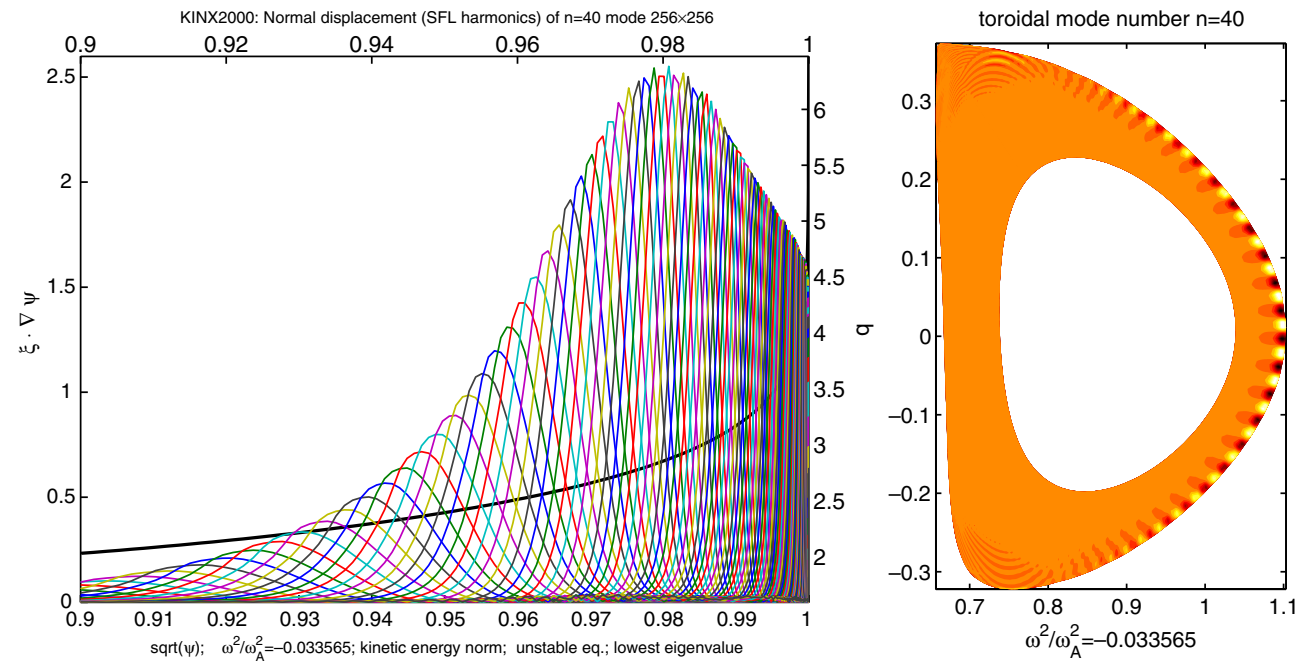

Figure 11. Ballooning dominated mode structure. Equilibrium corresponding to TCV \#8856 with the pedestal inside the plasma $\left(x_{0}=0.98, w=0.02, p^{\prime} / p_{c}^{\prime}=1.6 J_{\|} /\langle J\rangle=0.4\right)$. Toroidal wave number $n=40$.

\section{Conclusions}

- For fixed plasma boundary and pedestal shape the edge stability boundaries are close to each other in the parametric plane $\left(p^{\prime} / p_{c}^{\prime}, J_{\|} /\langle J\rangle\right)$ for modes with toroidal mode numbers $n$ following the scaling $w \times n \times q_{95}=$ const under variations of the pedestal width $w$ and the value of $q_{95}$.

- The value of $J_{\|} /\langle J\rangle \sim 1$ is a good approximation for the limit against edge current driven modes. The limit for pressure gradient $p^{\prime} / p_{c}^{\prime}$ set by coupled current driven kink/ballooning modes is sensitive to the shape of the plasma boundary. High values of triangularity $\delta>0.5$ combined with a sufficient elongation $E \gtrsim 2$ and/or cross-section squareness are favourable for the second stability access giving larger values of stable pressure gradients in the pedestal. 
- We note that $w \times n \times q_{95}=$ const corresponds to having approximately the same number of rational surfaces in the pedestal region for a given value of shear $S$. It could be sufficient to give the same leading order (in powers of $n$ ) contribution into the perturbed magnetic energy functional; fixing a point in the parametric plane $\left(p^{\prime} / p_{c}^{\prime}, J_{\|} /\langle J\rangle\right)$ leads to approximately the same driving terms in the potential energy functional and the same shear in the pedestal.

- We have studied pedestal profile shapes differing in the position of maximum $p^{\prime}$ and $J_{\|}$ with respect to the separatrix. The ratio of the edge current density to its maximum in the pedestal determines the coupling of external kink modes to ballooning modes. This affects the shape of the stability boundary in the parametric plane $\left(p^{\prime} / p_{c}^{\prime}, J_{\|} /\langle J\rangle\right)$ and in particular the access to the second stability region. Also, it affects the value of the constant in the $w \times n \times q_{95}=$ const scaling.

A more detailed analysis is required to compare these qualitative results with specific experimental observations. TCV can now measure edge profiles more accurately thanks to an upgrade of the Thomson scattering system [15]. The use of these measurements, with self-consistent equilibria including the edge bootstrap current, will be used for a further work dedicated to quantitative comparisons with experimental observations of the ELM's characteristics. In particular, the relation between the width, $q_{95}$ and the value $n$ of the most unstable mode, as well as the effect of shape on the stability limits, need to be compared specifically.

\section{Acknowledgments}

This work was partly supported by the Swiss National Science Foundation.

\section{Appendix A. Ballooning factor extraction in the case of x-point}

The ballooning factor extraction (BFX) is the use of the following displacement representation [14]:

$$
\begin{aligned}
& \xi=\tilde{\xi}(\psi, \theta) \exp \left(\text { in } \phi-\mathrm{i} n \int_{\theta_{0}}^{\theta} v(\psi, \theta) \mathrm{d} \theta\right), \\
& v(\psi, \theta)=\boldsymbol{B} \cdot \nabla \phi / \boldsymbol{B} \cdot \nabla \theta=-\sqrt{g} F / r^{2}, \\
& \quad \sqrt{g}=(\nabla \psi \times \nabla \theta \cdot \nabla \phi)^{-1}, \quad \boldsymbol{B}=\nabla \psi \times \nabla \phi+F(\psi) \nabla \phi .
\end{aligned}
$$

To ensure the displacement periodicity a jump condition should be set:

$$
\tilde{\xi}\left(\psi, \theta_{0}+\pi\right)=\exp \left(\mathrm{in} \int_{0}^{2 \pi} v(\psi, \theta) \mathrm{d} \theta\right) \tilde{\xi}\left(\psi, \theta_{0}-\pi\right)
$$

At the x-point $|\nabla \psi|=0$ and the value of $v$ goes to infinity. A straightforward way to treat the arising singularity is to match the position of the jump with the $\mathrm{x}$-point. It means that the phase in (A.1) goes to infinity only at the X-point and the singularity introduced by the BFX into the potential energy functional is the same as that at the $\mathrm{x}$-point in the original functional. In particular the $\psi$-derivative of $\xi_{\psi}=\xi \cdot \nabla \psi$ gives the following expression after the BFX transformation:

$$
\partial \tilde{\xi}_{\psi} / \partial \psi-\mathrm{i} n\left(\int_{\theta_{0}}^{\theta} \partial v / \partial \psi \mathrm{d} \theta\right) \tilde{\xi}_{\psi}
$$


The singularity in $\int_{\theta_{0}}^{\theta} v \mathrm{~d} \theta \sim q$ is logarithmic in $x=\psi-\psi_{s x}$ so the last term in (A.3) term remains of the same order in $x$ as the term $\alpha \xi_{\psi} / \alpha \psi$ due to the fact that $\xi_{\psi} \sim \sqrt{x}$ near the $\mathrm{X}$-point.

\section{Appendix B. Ballooning factor extraction with multiple jumps}

In the case of several $\mathrm{x}$-points at the boundary (for example double null up-down symmetric equilibrium) each of the $\mathrm{x}$-points should be matched with the BFX jump. The multiple jump option is also useful in increasing the efficiency of the BFX; the traditional choice of the jump at the high field side together with the jump at the X-point can significantly improve convergence. In the multiple jump option the integral in the phase of the BFX factor is chosen to be

$$
\int_{\theta_{j}}^{\theta} v(\psi, \theta) \mathrm{d} \theta, \quad \theta_{j}-\Delta \theta_{j} \leqslant \theta \leqslant \theta_{j}+\Delta \theta_{j}, \quad j=1, \ldots, J .
$$

The jumps are in the points $\theta_{j}+\Delta \theta_{j}$ and the integration ranges in $\theta$ cover the period such that $\theta_{j}+\Delta \theta_{j}=\theta_{j+1}-\Delta \theta_{j+1}, \quad j=1, \ldots, J-1$ and $\theta_{1}+\Delta \theta_{1}=\theta_{J}-\Delta \theta_{J}$.

To ensure the displacement continuity the following jump conditions are set:

$$
\tilde{\xi}\left(\psi, \theta_{j}+\Delta \theta_{j}\right)=\exp \left(\mathrm{i} n \int_{\theta_{j}}^{\theta_{j+1}} v(\psi, \theta) \mathrm{d} \theta\right) \tilde{\xi}\left(\psi, \theta_{j+1}-\Delta \theta_{j+1}\right)
$$

The implementation of the BFX in the KINX code is invariant against the choice of poloidal angle $\theta$. The jump lines $\theta=\theta_{j}+\Delta \theta_{j}$ are defined just by picking up certain grid points at the plasma boundary.

The proposed approach was validated for the equilibria with the x-point at the boundary and is a standard option in the current version of the KINX code for single axis tokamak and doublet cases.

\section{References}

[1] Connor J W et al 1998 Phys. Plasmas 52687

[2] Snyder P B et al 2002 Phys. Plasmas 92037

[3] Lortz D 1975 Nucl. Fusion 1549

[4] Wesson J A 1978 Nucl. Fusion 1897

[5] Medvedev S Yu, Degeling A, Martin Y, Sauter O and Villard L 2003 Edge kink/ballooning mode stability in TCV 30th EPS Conf. on Controlled Fusion and Plasma Physics (St. Petersburg, Russia, 7-11 July 2003) (ECA) vol 27A P-3.129

[6] Degtyarev L et al 1997 Comput. Phys. Comm. 10310

[7] Lao L L et al 2001 Nucl. Fusion 41295

[8] Snyder P B and Wilson H R 2003 Ideal magnetohydrodynamic constraints on the pedestal temperature in tokamaks Plasma Phys. Control. Fusion 451671

[9] Degeling A W et al 2003 Plasma Phys. Control. Fusion 451637

[10] Sauter O, Angioni C and Lin-Liu Y R 1999 Phys. Plasmas 62834

[11] Medvedev S.Yu et al 2004 Edge kink/ballooning mode stability scalings and ELM triggering in plasma with separatrix 31th EPS Conf. on Controlled Fusion and Plasma Physics (London, UK, 28 June-2 July 2004) (ECA) vol 28G P-2.147

[12] Ferron J R et al 2000 Phys. Plasmas 71976

[13] Moret J M et al 1997 Phys. Rev. Lett. 792057

[14] Gruber R et al 1981 Comput. Phys. Comm. 24363

[15] Alfier A et al 2005 Influence of ELMs on edge temperature and density profiles in TCV 32nd EPS Conf. on Plasma Physics (Tarragona, Spain, 27 June-1 July 2005) (ECA) vol 29C P-2.002 